\title{
Hypoglycemia with intensive insulin therapy after cardiac surgery: Predisposing factors and association with mortality
}

\author{
Sotiris C. Stamou, MD, PhD, ${ }^{a}$ Marcy Nussbaum, MS, ${ }^{\mathrm{b}}$ John D. Carew, PhD, MS, ${ }^{\mathrm{b}}$ Kelli Dunn, MD, ${ }^{\mathrm{c}}$ \\ Eric Skipper, MD, ${ }^{\mathrm{d}}$ Francis Robicsek, MD, PhD, ${ }^{\mathrm{d}}$ and Kevin W. Lobdell, $\mathrm{MD}^{\mathrm{d}}$
}

\begin{abstract}
Background: Intensive insulin therapy has become a major therapeutic target in cardiac surgery patients. It has been associated, however, with an increased risk of hypoglycemia compared with conventional insulin therapy. Our study sought to identify the factors predisposing to hypoglycemia with intensive insulin therapy and investigate its effect on early clinical outcomes after cardiac surgery.
\end{abstract}

\begin{abstract}
Methods: A concurrent cohort study of 2,538 consecutive patients undergoing cardiac surgery (coronary artery bypass grafting, valve, or bypass grafting and valve surgery) from January 2005 to March 2010 was carried out. Multivariable logistic regression analysis and propensity score matching were used (1) to identify the risk factors for developing hypoglycemia (blood glucose $<60 \mathrm{mg} / \mathrm{dL}$ ) after cardiac surgery and (2) to compare major morbidity, operative mortality, and actuarial survival between patients in whom hypoglycemia developed $(\mathrm{n}=77)$ and those in whom it did not $(\mathrm{n}=2461)$. The propensity score-adjusted sample included 61 patients in whom hypoglycemia developed and 305 patients in whom it did not (1 to 5 matching).
\end{abstract}

Results: Risk factors for hypoglycemia included female gender (odds ratio $[\mathrm{OR}]=2.3,95 \%$ confidence intervals $[\mathrm{CI}]=1.4-3.7 ; P<.001)$, diabetes $(\mathrm{OR}=2.8, \mathrm{CI}=1.7-4.5 ; P<.001)$, hemodialysis $(\mathrm{OR}=3.0, \mathrm{CI}=1.3-6.8$; $P=.009)$, intraoperative blood product transfusion $(\mathrm{OR}=2.0, \mathrm{CI}=1.2-3.4 ; P=.010)$, and earlier date of surgery (years of surgery, 2005-2007; $\mathrm{OR}=2.1, \mathrm{CI}=1.2-3.7 ; P=.007$ ). Hypoglycemia increased the risk for operative mortality in univariate (hypoglycemic $10 \%$ vs normoglycemic patients $2 \% ; P<.001$ ) but not in propensity scoreadjusted analysis $(\mathrm{OR}=2.5,0.9-6.7 ; P=.11)$. The propensity score-adjusted analysis demonstrated a significant increase in hemorrhage-related reexploration $(P=.048)$, pneumonia $(P<.001)$, reintubation $(P<.001)$, prolonged ventilatory support $(P<.001)$, hospital length of stay $(P<.001)$, and intensive care unit length of stay $(P<.001)$ for the hypoglycemic compared with normoglycemic patients. Five-year actuarial survival was similar in the compared patient groups (hypoglycemic $75 \%$ vs normoglycemic $75 \% ; P=.22$ ).

Conclusions: Hypoglycemia with intensive insulin therapy is independently associated with increased risk for respiratory complications and prolonged hospital and intensive care unit lengths of stay after cardiac surgery. In our study, hypoglycemia was not independently associated with increased risk of death. (J Thorac Cardiovasc Surg 2011;142:166-73)

Diabetes has been recognized as an independent risk factor for the development of coronary artery disease, ${ }^{1}$ which represents the most common cause of death in diabetic patients. ${ }^{2}$ Diabetes is also perceived as a risk factor for adverse outcomes after cardiac surgery. ${ }^{3-5}$ The Diabetes Mellitus Insulin-Glucose Infusion in Acute Myocardial

From the Department of Thoracic and Cardiovascular Surgery, ${ }^{\mathrm{a}}$ Missouri Baptist Medical Center, St Louis, Mo; the Dickson Institute for Health Studies, ${ }^{\mathrm{b}}$ Carolinas Health Care System, Charlotte, NC; the Department of Medicine, ${ }^{\mathrm{c}}$ Carolinas Health Care System, Charlotte, NC; and the Sanger Heart and Vascular Institute, ${ }^{d}$ Carolinas Health Care System, Charlotte, NC.

Disclosures: Authors have nothing to disclose with regard to commercial support.

Received for publication June 15, 2010; revisions received Aug 7, 2010; accepted for publication Sept 12, 2010; available ahead of print March 14, 2011.

Address for reprints: Kevin W. Lobdell, MD, 1000 Blythe Blvd, Charlotte, NC 28203 (E-mail: kevin.lobdell@carolinashealthcare.org) or Address for correspondence: Sotiris C. Stamou, MD, 1315 Whispering Pines Dr, Apt L, St Louis, MO 63146. (E-mail: cvsisfun@hotmail.com).

$0022-5223 / \$ 36.00$

Copyright (c) 2011 by The American Association for Thoracic Surgery doi:10.1016/j.jtcvs.2010.09.064
Infarction (DIGAMI) study reported that glycemic control by insulin infusions provided better long-term survival in diabetic patients having experienced myocardial infarction. ${ }^{6}$ Other studies suggested that strict glucose control can improve outcomes in diabetic patients undergoing myocardial revascularization. ${ }^{7,8}$

Barriers to widespread adoption of tight glucose control include the increased risk of severe hypoglycemia, ${ }^{9}$ the difficulty in achieving normoglycemia in critically ill patients, ${ }^{10,11}$ and the increased resources that would be required. ${ }^{12}$ The Normoglycemia in Intensive Care Evaluation-Survival Using Glucose Algorithm Regulation (NICE-SUGAR) trial demonstrated that intensive glucose control increased mortality among adults in the intensive care unit (ICU) and that a blood glucose target of $180 \mathrm{mg} /$ $\mathrm{dL}$ or less resulted in lower mortality than did a target of 81 to $108 \mathrm{mg} / \mathrm{dL} .^{13}$ The aim of our study was to identify the factors predisposing to hypoglycemia with intensive insulin therapy and to investigate its effect on clinical outcomes after cardiac surgery. 


\begin{tabular}{|c|c|}
\hline \multicolumn{2}{|c|}{ Abbreviations and Acronyms } \\
\hline \multicolumn{2}{|c|}{ 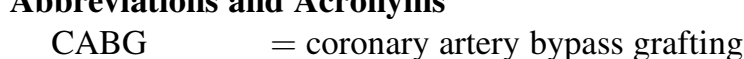 } \\
\hline $\mathrm{CI}$ & $=$ confidence intervals \\
\hline DIGAMI & $\begin{aligned}= & \text { Diabetes Mellitus Insulin- } \\
& \text { Glucose Infusion in Acute } \\
& \text { Myocardial Infarction }\end{aligned}$ \\
\hline ICU & $=$ intensive care unit \\
\hline IV & $=$ intravenous \\
\hline HIPAA & $\begin{aligned}= & \text { Health Insurance Portability } \\
& \text { and Accountability Act of } 1996\end{aligned}$ \\
\hline \multicolumn{2}{|c|}{ NICE-SUGAR $=$ Normoglycemia in Intensive } \\
\hline & Care Evaluation-Survival \\
\hline & Using Glucose Algorithm \\
\hline & Regulation \\
\hline OR & $=$ odds ratio \\
\hline
\end{tabular}

\section{PATIENTS AND METHODS \\ Patients}

The database of the Division of Cardiothoracic Surgery at the Sanger Heart and Vascular Institute was queried and identified 2538 consecutive patients undergoing cardiac surgery (coronary artery bypass grafting [CABG], valve, or CABG and valve surgery) from January 2005 to March 2010; with our glycemic control protocol, hypoglycemia developed in 77 of those patients. All patients undergoing cardiac surgery at our institution have been receiving a protocol for insulin administration for glycemic control. The operations were performed by the same group of cardiac surgeons for the study period. Baseline demographics, procedural data, and perioperative outcomes were recorded and entered prospectively in a prespecified database by dedicated data-coordinating personnel.

\section{Study Design and Conduct}

This is a retrospective cohort study of prospectively collected data from consecutive patients who underwent CABG, valve, or CABG and valve surgery at the Sanger Heart and Vascular Institute. Study approval was sought and obtained from the Institutional Review Board. Patient confidentiality was maintained at all times, consistent with the Health Insurance Portability and Accountability Act of 1996 (HIPAA) regulations.

\section{Glycemic Control Protocol}

All patients undergoing cardiac surgery at our institution have been receiving a protocol to standardize the titration of insulin therapy during the postoperative period. Intraoperative blood glucose levels were maintained below $200 \mathrm{mg} / \mathrm{dL}$ with intravenous (IV) regular insulin. Glucose levels were checked on all patients on admission to the ICU. Diabetic patients were started on IV insulin if the serum glucose was greater than 130 $\mathrm{gm} / \mathrm{dL}$. Patients without diabetes were started on IV insulin if the serum glucose was greater than $150 \mathrm{gm} / \mathrm{dL}$. Euglycemia was managed with a computerized software system (EndoTool glucose management system; Appendix 1). This software calculates the dose of IV insulin needed. It also actively models and adapts to individual patient responses to IV insulineven those with fluctuating insulin requirements (http://www.hospira.com/ Products/endotool.aspx). ${ }^{14} \mathrm{IV}$ insulin was continued until the patient was able to eat, whereupon the computerized algorithm generated a subcutaneous insulin regimen and the IV insulin was discontinued. Our glycemic control and intensive insulin therapy protocol order set is depicted in Appendix 1.

\section{Definitions}

The Society of Thoracic Surgeons' national cardiac surgery database definitions were used for this study. Hypoglycemia after cardiac surgery was defined as blood glucose less than $60 \mathrm{mg} / \mathrm{dL}$. Previous cerebrovascular accident was defined as history of central neurologic deficit persisting for more than 24 hours. Chronic renal insufficiency was defined as a serum creatinine value of $2.0 \mathrm{mg} / \mathrm{dL}$ or more. Diabetes was defined as a history of diabetes mellitus, regardless of duration of disease or need for oral agents or insulin. Depressed ejection fraction was defined as an ejection fraction less than $40 \%$. Prolonged ventilatory support was defined as pulmonary insufficiency requiring ventilatory support for more than 24 hours postoperatively. Postoperative stroke was defined as any new major (type I) neurologic deficit presenting in-hospital and persisting for more than 72 hours. ${ }^{15}$ Acute renal failure was defined as one or both of the following: (1) an increase in the serum creatinine to more than $2.0 \mathrm{mg} / \mathrm{dL}$ and/or a greater than 2-fold increase in the most recent preoperative creatinine level or (2) a new requirement for dialysis postoperatively. Prolonged hospital length of stay was defined as hospital stay greater than 14 days. Operative mortality includes both (1) all deaths occurring during the hospitalization in which the operation was performed (even if death occurred after 30 days from the operation) and (2) those deaths occurring after discharge from the hospital but within 30 days of the procedure.

\section{Data Analysis}

Univariate analysis. Univariate comparisons of preoperative, operative, and postoperative variables were performed between hypoglycemic $(n=77)$ and normoglycemic patients $(n=2461)$. Continuous variables were summarized using medians and ranges; frequencies and percentages were reported for categorical variables. Continuous variables were tested using the Kruskal-Wallis test for association, and categorical variables were assessed by the $\chi^{2}$ or Fisher's exact test, depending on the distribution of the data. A $P$ value of $<.05$ was considered statistically significant. The Bonferroni correction was used on the postoperative outcomes $(n=10)$. The $P$ value of significance was determined to be $P<.005$ (10 tests: $.05 /$ $10=.005) .{ }^{16}$

Multivariable analysis. Multivariable logistic regression analysis was used to identify the risk factors for the development of hypoglycemia and operative mortality after cardiac surgery. Predictors were chosen for model inclusion if the univariate associations with the outcomes were $P<.1$. Backward stepwise elimination was performed to keep predictors at an alpha level $<.05$ for the final model.

Propensity score model. Propensity score adjustment was used to correct for imbalances between groups at baseline and compare the postoperative outcomes between hypoglycemic and normoglycemic patients. ${ }^{17,18}$ A logistic regression model was fit where the hypoglycemia group was the outcome and baseline characteristics $(P<.05)$ from the bivariate analysis were the covariates. Propensity scores were generated for each patient and used to match hypoglycemic and normoglycemic patients in a nearest neighbor fashion. The propensity score-adjusted sample included 61 patients in whom hypoglycemia developed and 305 patients who were normoglycemic ( 1 to 5 matching). One to 5 matching was chosen in an effort to improve efficiency in parameter estimation by increasing the sample size and subsequent power. Inasmuch as there are far more controls (nonhypoglycemic) than hypoglycemic patients, multiple controls may be selected for each hypoglycemic patient. Using 5 controls instead of 4 increased the efficiency by $4 \% .{ }^{19}$

\section{Survival Analysis}

Kaplan-Meier survival estimates were calculated for the propensity score-adjusted hypoglycemic patients and compared with the normoglycemic patients using a log-rank test.

All analyses were conducted using SAS version 9.2 (SAS Institute, Inc, Cary, NC). 
TABLE 1. Distribution of preoperative variables in hypoglycemic and normoglycemic patients (univariate analysis)

\begin{tabular}{|c|c|c|c|}
\hline & $\begin{array}{c}\text { Hypoglycemic } \\
(\mathbf{n}=77)\end{array}$ & $\begin{array}{l}\text { Normoglycemic } \\
\quad(n=2461)\end{array}$ & $\begin{array}{c}P \\
\text { value }\end{array}$ \\
\hline Female gender & $39(51 \%)$ & $643(26 \%)$ & $<.001$ \\
\hline Diabetes & $49(64 \%)$ & $845(34 \%)$ & $<.001$ \\
\hline Congestive heart failure & $22(29 \%)$ & $343(14 \%)$ & $<.001$ \\
\hline Age $>75 y$ & $12(16 \%)$ & $353(14 \%)$ & .76 \\
\hline $\begin{array}{l}\text { Body mass index } \\
\text { (median/range) }\end{array}$ & $29(17-48)$ & $28(13-85)$ & .87 \\
\hline Hypertension & $66(86 \%)$ & $1949(79 \%)$ & .16 \\
\hline Chronic renal insufficiency & $11(14 \%)$ & $97(4 \%)$ & $<.001$ \\
\hline Ejection fraction $<40$ & $25(33 \%)$ & $483(20 \%)$ & .006 \\
\hline $\begin{array}{l}\text { Ejection fraction } \\
\text { (median/range) }\end{array}$ & $47(19-70)$ & $55(5-86)$ & .016 \\
\hline \multicolumn{4}{|l|}{$\begin{array}{l}\text { Chronic Obstructive } \\
\text { pulmonary disease }\end{array}$} \\
\hline Moderate/severe & $3(4 \%)$ & $139(6 \%)$ & .80 \\
\hline Hemodialysis & $8(10 \%)$ & $58(3 \%)$ & $<.001$ \\
\hline Acute myocardial infarction & $35(46 \%)$ & $952(39 \%)$ & .23 \\
\hline History of stroke & $9(12 \%)$ & $228(9 \%)$ & .47 \\
\hline Unstable angina & $1(1 \%)$ & $196(8 \%)$ & .031 \\
\hline \multicolumn{4}{|l|}{ No. of diseased vessels } \\
\hline One & $13(17 \%)$ & $202(8 \%)$ & .052 \\
\hline Two & $17(22 \%)$ & $642(26 \%)$ & \\
\hline Three & $34(44 \%)$ & $1096(45 \%)$ & \\
\hline None & $13(17 \%)$ & $520(21 \%)$ & \\
\hline \multicolumn{4}{|l|}{ Case priority } \\
\hline Elective & $23(30 \%)$ & $942(38 \%)$ & .047 \\
\hline Urgent & $45(58 \%)$ & $139(6 \%)$ & \\
\hline Emergency & $9(12 \%)$ & $1380(56 \%)$ & \\
\hline \multicolumn{4}{|l|}{ Year of surgery } \\
\hline 2005 & $14(18 \%)$ & $424(17 \%)$ & $<.001$ \\
\hline 2006 & $14(18 \%)$ & $470(19 \%)$ & \\
\hline 2007 & $31(40 \%)$ & $510(21 \%)$ & \\
\hline 2008 & $9(12 \%)$ & $460(19 \%)$ & \\
\hline 2009 & $4(5 \%)$ & $480(20 \%)$ & \\
\hline 2010 & $5(7 \%)$ & $117(5 \%)$ & \\
\hline
\end{tabular}

\section{RESULTS}

\section{Univariate Analysis}

Preoperative characteristics. Preoperative patient characteristics are shown in Table 1. Hypoglycemic patients were more likely to be female, have diabetes mellitus, congestive heart failure, chronic renal insufficiency, ejection fraction less than $40 \%$, be on hemodialysis, and have surgery during the earlier years of the study (2005-2007). Normoglycemic patients were more likely to have unstable angina than were hypoglycemic patients.

Operative and postoperative characteristics. Operative and postoperative patient characteristics are presented in Table 2. Because of the Bonferroni correction for postoperative outcomes, a $P$ value was considered statistically significant if it was less than .005. Hypoglycemic patients were more likely to receive intraoperative blood products
TABLE 2. Distribution of intraoperative and postoperative variables in hypoglycemic and normoglycemic patients (univariate analysis)

\begin{tabular}{lccc}
\hline & $\begin{array}{c}\text { Hypoglycemic } \\
(\mathbf{n}=\mathbf{7 7})\end{array}$ & $\begin{array}{c}\text { Normoglycemic } \\
(\mathbf{n}=\mathbf{2 4 6 1})\end{array}$ & $\begin{array}{c}\boldsymbol{P} \\
\text { value }\end{array}$ \\
\hline Intraoperative variables & & & \\
CABG & $60(78 \%)$ & $1896(77 \%)$ & .86 \\
$\quad$ Valve surgery & $28(36 \%)$ & $756(31 \%)$ & .29 \\
Intraoperative blood & $51(66 \%)$ & $910(37 \%)$ & $<.001$ \\
$\quad$ transfusion & & & \\
Crossclamp time & $66(19-177)$ & $64(15-217)$ & .68 \\
$\quad$ (median, range) & & & \\
Crossclamp time $>2$ h & $8(12 \%)$ & $92(5 \%)$ & .018 \\
CPB time (median, range) & $104(16-263)$ & $98(23-318)$ & .39 \\
CPB time $>$ 2 h & $26(38 \%)$ & $518(26 \%)$ & .038 \\
Off-pump CABG & $68(88 \%)$ & $1962(80 \%)$ & .064 \\
Postoperative variables* & & & \\
Hemorrhage-related & $11(14 \%)$ & $121(4.9 \%)$ & .002 \\
$\quad$ reexploration & & & \\
Prolonged ventilatory & $30(39 \%)$ & $250(10.2 \%)$ & $<.001$ \\
$\quad$ support (>24 h) & & & \\
Reintubation & $19(25 \%)$ & $134(5.4 \%)$ & $<.001$ \\
Pneumonia & $13(17 \%)$ & $86(3.5 \%)$ & $<.001$ \\
Acute renal failure & $11(14 \%)$ & $101(4.1 \%)$ & $<.001$ \\
Hemodialysis & $4(5 \%)$ & $29(1.2 \%)$ & .016 \\
Stroke & $8(10 \%)$ & $34(1.4 \%)$ & $<.001$ \\
Hospital LOS, d & $14(4-76)$ & $9(1-162)$ & $<.001$ \\
$\quad$ (median, range) & & & \\
ICU LOS, d (median, range) & $4.9(1-71)$ & $1.7(0-105)$ & $<.001$ \\
Operative mortality & $8(10 \%)$ & $60(2 \%)$ & $<.001$ \\
\hline CABG, Coronary artery bypass grafting; $C P B$, cardiopulmonary bypass; LOS, length \\
of stay; ICU, intensive care unit. *Bonferroni correction: 10 tests-.05/10= & .005.
\end{tabular}

than were normoglycemic patients. Hypoglycemic patients had a higher risk for operative mortality, hemorrhagerelated reexploration, acute renal failure, stroke, reintubation, prolonged ventilatory support, pneumonia, and prolonged hospital and ICU lengths of stay than were normoglycemic patients. Diabetes was associated with a similar operative mortality both in normoglycemic patients (diabetics $3 \%$ vs nondiabetics $2 \% ; P=.23$ ) and in hypoglycemic patients (diabetics $14 \%$ vs nondiabetics $4 \% ; P=.25)$. Operative mortality was similar in patients with blood glucose levels less than $40 \mathrm{mg} / \mathrm{dL}$ versus those with blood glucose levels of 61 to $80 \mathrm{mg} / \mathrm{dL}$ ( $23 \%$ vs $8 \%$; $P=.13$ ). Diabetes did not increase the risk of mortality in either hypoglycemic or normoglycemic patients. Hypoglycemia rates were lower in the latter half of the study period (4\% for $2005-007$ vs. $2 \%$ for $2008-2010 ; P=.007$ ). Fifty-nine patients had 1 hypoglycemic episode, 13 had 2 episodes, 3 had 3 episodes, 1 had 5 episodes, and 1 had 8 hypoglycemic episodes. Patients who had more than 1 episode of hypoglycemia had similar operative mortality (single hypoglycemic episode $9 \%$ vs multiple episodes $17 \% ; P=.38$ ), stroke (single hypoglycemic episode $10 \%$ vs multiple episodes $11 \% ; P>.99$ ), and prolonged ventilatory support (single hypoglycemic episode 
$34 \%$ vs multiple episodes $56 \% ; P=.17$ ) compared with those who had multiple hypoglycemic episodes. ICU length of stay was more abbreviated, however, in patients with single compared with multiple episodes of hypoglycemia (single hypoglycemic episode median ICU stay 4.5 days, range $=1-57$ days, vs multiple episodes median ICU stay 8 days, range $=1-71$ days; $P=.03$ ). Hospital length of stay, however, was similar between the patients who had a single hypoglycemic episodes versus those who had multiple episodes (single hypoglycemic episode median hospital stay 14 days, range $=4-65$ days, vs multiple episodes median hospital stay 14 days, range $=5-76$ days; $P=.49$ ).

\section{Multivariable Logistic Regression Analysis}

Female gender (odds ratio $[\mathrm{OR}]=2.3,95 \%$ confidence intervals $[\mathrm{CI}]=1.4-3.7 ; P<.001)$, diabetes $(\mathrm{OR}=2.8$, $\mathrm{CI}=1.7-4.5 ; P<.001)$, hemodialysis $(\mathrm{OR}=3.0$, $\mathrm{CI}=1.3-6.8 ; P=.009)$, intraoperative blood product transfusion $(\mathrm{OR}=2.0, \mathrm{CI}=1.2-3.4 ; P=.010)$, and earlier date of surgery (year of surgery 2005-2007 OR $=2.1$, $\mathrm{CI}=1.2-3.7 ; P=.007)$ emerged as independent predictors of hypoglycemia after cardiac surgery. The $\mathrm{C}$ statistic for the model was $74 \%$, which indicates a good fit. Hypoglycemia was also identified as an independent predictor of mortality in the multivariate logistic regression analysis $(\mathrm{OR}=2.7$, $95 \% \mathrm{CI}=1.1-6.3 ; P=.027, \mathrm{C}$ statistic $=76 \%$; Table 3 ). Propensity score analysis. The ability of the propensity score to effectively balance the groups at baseline was confirmed by showing that the variables put into the propensity score were no longer significant in the matched sample (Table 4). Hence, the groups were more balanced for the subsequent testing of outcomes in the matched samples. Results of the propensity score-adjusted analysis are depicted in Table 5. Another Bonferroni correction was done so that $P$ values $<.005$ were considered statistically significant. The propensity score-adjusted OR demonstrated a significant increase in hemorrhage-related reexploration $(P=.0048)$, pneumonia $(P<.001)$, reintubation $(P<.001)$, prolonged ventilatory support $(P<.001)$, and prolonged hospital $(P<.001)$ and ICU lengths of stay

TABLE 3. Multivariable logistic regression analysis model of operative mortality

\begin{tabular}{lrrrrr}
\hline & Beta & SD & OR & 95\% CI & $\boldsymbol{P}$ value \\
\hline Hypoglycemia & .49 & .22 & 2.7 & $1.1-6.3$ & .027 \\
Diabetes & .31 & .14 & 1.9 & $1.1-3.2$ & .021 \\
Congestive heart failure & .45 & .14 & 2.4 & $1.4-4.3$ & .002 \\
Age $>75$ y & .54 & .14 & 3.0 & $1.7-5.1$ & $<.001$ \\
Valve surgery & .32 & .14 & 1.9 & $1.1-3.4$ & .023 \\
Case priority & & & & & \\
$\quad$ Elective vs urgent & -.61 & .21 & 0.8 & $0.4-1.5$ & .003 \\
$\quad$ Emergency vs urgent & 1.02 & .23 & 4.2 & $2.1-8.3$ & $<.001$ \\
\hline SD, Stand din
\end{tabular}

$S D$, Standard deviation; $O R$, odds ratio; $C I$, confidence intervals. C statistic $=76 \%$
TABLE 4. $P$ values before and after propensity score matching

\begin{tabular}{lrr}
\hline & Before & After \\
\hline Female gender & $<.001$ & .71 \\
Diabetes & $<.001$ & .45 \\
Congestive heart failure & .006 & .82 \\
Chronic renal insufficiency & .002 & .61 \\
Ejection fraction $<40$ & .006 & .88 \\
Chronic obstructive pulmonary disease & $<.001$ & .63 \\
Hemodialysis & $<.001$ & .40 \\
Unstable angina & .031 & $>.99$ \\
Status & .047 & .80 \\
Year of surgery & $<.001$ & .81 \\
\hline
\end{tabular}

$(P<.001)$ for the hypoglycemic compared with the normoglycemic patients. Propensity score adjustment also showed a similar mortality for hypoglycemic compared with normoglycemic patients $(P=.11)$.

Survival analysis. Kaplan-Meier survival estimates are presented in Figure 1. There was no difference in the actuarial 5-year survival between hypoglycemic and normoglycemic patients in the propensity score-matched population. Specifically, actuarial 5-year survival was $75 \%$ for hypoglycemic patients versus $75 \%$ for normoglycemic patients (Figure 1).

\section{DISCUSSION}

Intensive insulin therapy has become a standard modality in patients undergoing cardiac surgery. Previous studies reported that strict glycemic control with intensive insulin therapy may improve outcomes after cardiac surgery, ${ }^{20}$ and others reported increased mortality with intensive insulin therapy among adults in the ICU. ${ }^{13,21}$ Specifically according to the NICE-SUGAR study, intensive glucose control with a blood glucose target of $180 \mathrm{mg} / \mathrm{dL}$ resulted in higher mortality than did a target blood glucose of 180 $\mathrm{mg} / \mathrm{dL}$ or less. Among other risks, intensive insulin therapy has been associated with increased risk of severe hypoglycemia. Randomized controlled trials of intensive insulin therapy reported variable frequency of hypoglycemic episodes ranging between $5 \%$ and $32 \%$ of patients treated with intensive insulin therapy compared with $0.8 \%$ to $5 \%$ in patients treated with conventional insulin therapy protocols. ${ }^{22-24}$ Differences in hypoglycemia rates with intensive insulin therapy may be related to different protocols, different proportions of diabetic patients, and different patient populations. The effect of hypoglycemia after cardiac surgery has not been thoroughly investigated. Our study identified the factors predisposing to hypoglycemia with intensive insulin therapy and evaluated the effect of hypoglycemia on clinical outcomes after cardiac surgery.

\section{Principal Findings}

Hypoglycemic patients experience greater morbidity and similar operative mortality compared with normoglycemic 
TABLE 5. Adjusted by propensity score ORs for early hospital outcomes in normoglycemic and hypoglycemic patient groups

\begin{tabular}{|c|c|c|c|c|c|}
\hline & $\begin{array}{c}\text { Hypoglycemic } \\
(\mathrm{n}=61)\end{array}$ & $\begin{array}{l}\text { Normoglycemic } \\
\quad(\mathbf{n}=\mathbf{3 0 5})\end{array}$ & OR & $95 \%$ CI & $\begin{array}{c}P \\
\text { value }\end{array}$ \\
\hline Hemorrhage-related reexploration & $10(16 \%)$ & $16(5 \%)$ & 3.5 & $1.5-8.2$ & .0048 \\
\hline Acute renal failure & $10(16 \%)$ & $20(7 \%)$ & 2.8 & $1.2-6.3$ & .011 \\
\hline Hemodialysis & $4(7 \%)$ & $6(2 \%)$ & 3.5 & $1.0-12.8$ & .067 \\
\hline Stroke & $6(10 \%)$ & $9(3 \%)$ & 3.6 & $1.2-10.5$ & .025 \\
\hline Hospital LOS, d (median, range) & $20(4-76)$ & $9(3-134)$ & NA & & $<.001$ \\
\hline ICU LOS, d (median, range) & $5(1-71)$ & $2(0-77)$ & NA & & $<.001$ \\
\hline Reintubation & $14(23 \%)$ & $19(6 \%)$ & 4.5 & $2.1-9.6$ & $<.001$ \\
\hline Prolonged ventilatory support (>24 h) & $23(38 \%)$ & $35(12 \%)$ & 4.7 & $2.5-8.7$ & $<.001$ \\
\hline Pneumonia & $10(16 \%)$ & $9(3 \%)$ & 6.5 & $2.5-16.7$ & $<.001$ \\
\hline Operative mortality & $6(10 \%)$ & $13(4 \%)$ & 2.5 & $0.9-6.7$ & .11 \\
\hline
\end{tabular}

$O R$, Odds ratio; $C I$, confidence intervals; $L O S$, length of stay; $I C U$, intensive care unit; $N A$, not available. Bonferroni correction: 10 tests $-.05 / 10=.005$.

patients after cardiac surgery, after adjusting for imbalances in the preoperative risk factors and using a stringent testing criterion (the Bonferroni correction), a finding that agrees with previous studies. ${ }^{24}$ When adjusted for potential confounders, hypoglycemia was not associated with increased mortality, which contradicts previous studies. Specifically, according to the NICE-SUGAR trial, intensive glucose control increased mortality among adults in the ICU, and a blood glucose target of $180 \mathrm{mg} / \mathrm{dL}$ or less resulted in lower mortality than did a target of 81 to $108 \mathrm{mg} / \mathrm{dL} .{ }^{13}$ Similarly, Hermanides and associates ${ }^{21}$ demonstrated an increased mortality with blood glucose levels below $45 \mathrm{mg} / \mathrm{dL}$ in the ICU. However, these 2 studies did not focus on the cardiac surgery population and used different definitions for hypoglycemia than did our study.

\section{Hypoglycemia and Postoperative Complications}

We demonstrated that hypoglycemia was associated with a higher rate of hemorrhage-related reexploration, pro- longed ventilatory support, reintubation, pneumonia, and prolonged hospital and ICU lengths of stay. The surgical stress and the adrenergic response associated with it may explain the higher frequency of hypoglycemic episodes in patients who had hemorrhage-related reexploration. Hypoglycemia may contribute to postoperative stroke by inducing neuroglycopenia with neuronal cell loss and hypoglycemic coma, especially after hyperglycemic reperfusion. $^{21}$ Furthermore, hypoglycemia might provoke ischemia in pre-existing vascular disease in patients with diabetes $^{25}$ and it has also been shown to induce platelet activation and inflammation in an experimental setting. ${ }^{26}$ Hypoglycemia could thus contribute to acute renal failure, respiratory complications, and prolonged hospital length of stay by aggravating overall illness. ${ }^{21}$ Hypoglycemia was more frequent in diabetic patients, possibly because of a wider fluctuation of blood glucose levels, a more unpredictable response to the infused insulin, as well as a higher interindividual variability in the diabetic versus the

\section{Freedom from Death at 5 Years}

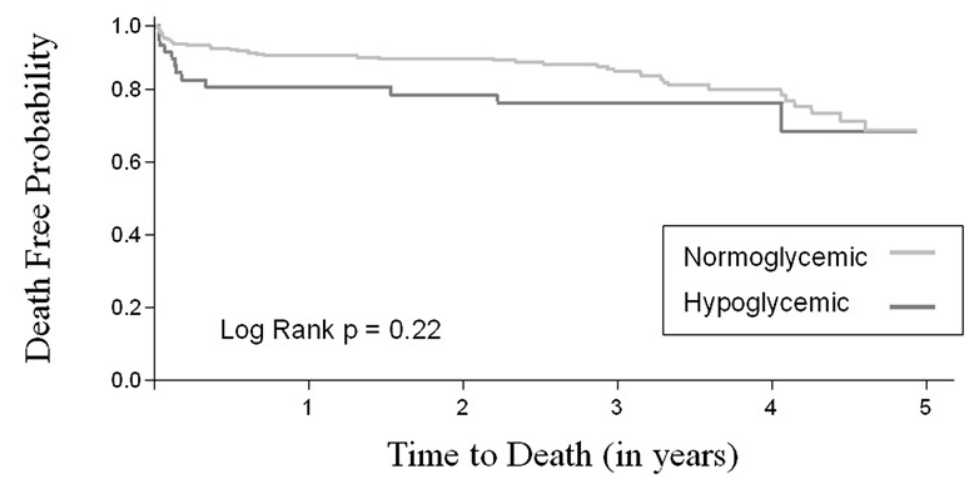

$\begin{array}{lclcllc}\text { Number at Risk } & 30 \text { days } & 1 \text { year } & 2 \text { years } & 3 \text { years } & 4 \text { years } & 5 \text { years } \\ \text { Normoglycemic } & 295(97 \%) & 283(93 \%) & 281(92 \%) & 276(91 \%) & 259(85 \%) & 228(75 \%) \\ \text { Hypoglycemic } & 57(93 \%) & 52(85 \%) & 51(84 \%) & 50(82 \%) & 50(82 \%) & 46(75 \%)\end{array}$

FIGURE 1. Freedom from death at 5 years. Kaplan-Meier survival estimates in the propensity score-adjusted patient groups. $P$ value, calculated with the $\log$-rank test, is reported. 
nondiabetic patients. ${ }^{27}$ The lower rate of hypoglycemia in the latter half of the study period may be attributed to employment of a rigorous quality improvement protocol in our institution that also resulted in a decrease in operative mortality, as $\mathrm{we}^{28}$ previously reported.

Single hypoglycemic episodes were associated with similar mortality and postoperative morbidity but a more abbreviated ICU length of stay compared with those in patients who had multiple hypoglycemic episodes.

\section{Actuarial Survival}

Our study demonstrated a similar actuarial 5-year survival in hypoglycemic and normoglycemic patients ( $\log -$ rank $P=.22$, Figure 1$)$. As depicted in Figure 1, there was an initial survival difference secondary to a higher early mortality for the hypoglycemic compared with the normoglycemic patients; however, at 5 years the 2 curves touch each other, reflecting a similar 5-year survival.

\section{Clinical Implications}

In this study we used a real-world unselected cohort of patients with prospectively acquired data by dedicated data management personnel to compare early hospital outcomes between hypoglycemic and normoglycemic patient groups and to evaluate the risk factors for hypoglycemia after cardiac surgery. On the basis of our results, hypoglycemic patients had a higher operative mortality as well as a higher rate of hemorrhage-related reexploration, prolonged ventilatory support, reintubation, pneumonia, and prolonged hospital and ICU lengths of stay than normoglycemic patients in univariate analysis. After propensity score adjustment, operative mortality was similar, but the respiratory complications and hemorrhage-related reexploration rates were higher and hospital and ICU lengths of stay were more prolonged in the hypoglycemic patients than in the normoglycemic patients. Our study also showed a similar actuarial 5-year survival for hypoglycemic compared with normoglycemic patients. Among the factors found to increase the risk of hypoglycemia (female gender, diabetes, hemodialysis, intraoperative blood transfusions) after cardiac surgery, only intraoperative blood transfusion is amenable to therapeutic intervention. A higher target level of blood glucose in the postoperative period may decrease the frequency of hypoglycemic episodes and thus reduce the rates of complications associated with it. A more focused and individualized approach may be warranted for diabetics, women, and patients with renal failure and should stimulate future studies. Future studies may also be warranted to evaluate the optimal goal of blood glucose levels after cardiac surgery, at which improved outcomes are achieved without significant hypoglycemic risk. Prospective randomized studies may help to better define strategies, benefits, and risks of intensive insulin therapy.

\section{STUDY LIMITATIONS}

Limitations of this study include all those inherent in any retrospective, single-institution analysis. This was a nonrandomized study in which unmeasured patient- or procedure-related variables may have influenced the study results. Furthermore, this investigation was conducted at a large tertiary referral center, and the results may not be broadly representative of community practice. Another limitation of our study was the small number of the hypoglycemic patient group, which may have prevented us from demonstrating a difference in mortality secondary to a type II error. There are also several known and unknown variables such as steroid use, discontinuation of catecholamines, and others that were not adjusted for in the propensity score-adjusted multivariable analysis. Our study did not compare different insulin therapy protocols to identify one that best achieves the glycemic targets associated with the lowest hypoglycemia risk. Evaluation of the effects of hypoglycemia variability and hypoglycemia temporal trends on the postoperative outcome was beyond the scope of our study. Moreover, the level of $60 \mathrm{mg} / \mathrm{dL}$ is not necessarily an accurate marker of hypoglycemia inasmuch as hormonal imbalance may present at even higher levels of blood glucose. The ranges, averages, and median glucose values were not available to us during the study period and will be the focus of future investigations by our institution. Among the strengths of this study are the large cohort of patients, the prospective entry of all data elements into a cardiac surgical research database with strict definitions, and analysis of data performed with appropriately risk-adjusted statistical models to adjust for differences in preoperative risk factors.

In conclusion, hypoglycemic patients had a similar operative mortality and 5-year actuarial survival and a higher rate of postoperative respiratory complications and prolonged hospital and ICU lengths of stay than did normoglycemic patients. Actuarial 5-year survival was similar in the 2 patient groups.

\section{References}

1. Kannel WB. McGee DL.Diabetes and cardiovascular disease. The Framingham study. JAMA. 1979;241:2035-8.

2. Bonow RO. Gheorghiade M.The diabetes epidemic: a national and global crisis. Am J Med. 2004;116(Suppl 5A):2S-10S.

3. Edwards FH, Grover FL, Shroyer AL, Schwartz M, Bero J. The Society of Thoracic Surgeons National Cardiac Surgery Database: current risk assessment Ann Thorac Surg. 1997;63:903-8

4. Thourani VH, Weintraub WS, Stein B, Gebhart SS, Craver JM, Jones EL, et al. Influence of diabetes mellitus on early and late outcome after coronary artery bypass grafting. Ann Thorac Surg. 1999;67:1045-52.

5. Barsness GW, Peterson ED, Ohman EM, Nelson CL, DeLong ER, Reves JG et al. Relationship between diabetes mellitus and long-term survival after coronary bypass and angioplasty. Circulation. 1997;96:2551-6.

6. Malmberg K, Rydén L, Efendic S, Herlitz J, Nicol P, Waldenström A, et al. Randomized trial of insulin-glucose infusion followed by subcutaneous in sulin treatment in diabetic patients with acute myocardial infarction (DIGAMI study): effects on mortality at 1 year. J Am Coll Cardiol. 1995; 26:57-65 
7. Furnary AP, Gao G, Grunkemeier GL, Wu Y, Zerr KJ, Bookin SO, et al. Continuous insulin infusion reduces mortality in patients with diabetes undergoing coronary artery bypass grafting. J Thorac Cardiovasc Surg. 2003;125:1007-21.

8. Lazar HL, Chipkin SR, Fitzgerald CA, Bao Y, Cabral H, Apstein CS. Tight glycemic control in diabetic coronary artery bypass graft patients improves perioperative outcomes and decreases recurrent ischemic events. Circulation. 2004; 109:1497-502.

9. Wiener RS, Wiener DC, Larson RJ. Benefits and risks of tight glucose control in critically ill adults: a meta-analysis. JAMA. 2008;300:933-44.

10. Shulman R, Finney SJ, O'Sullivan C, Glynne PA, Greene R. Improvement in glycemic control and outcome corresponding to intensive insulin therapy protocol development. J Diabetes Sci Technol. 2008;2:392-401.

11. Chase JG, Shaw GM. Is there more to glycaemic control than meets the eye? Crit Care. 2007;11:160.

12. Aragon D. Evaluation of nursing work effort and perceptions about blood glucose testing in tight glycemic control. Am J Crit Care. 2006;15:370-7.

13. NICE-SUGAR Study Investigators, Finfer S, Chittock DR, Su SY, Blair D, Foster D, Dhingra V, et al. Intensive versus conventional glucose control in critically ill patients. N Engl J Med. 2009;360:1283-97.

14. Saager L, Collins GL, Burnside B, Tymkew H, Zhang L, Jacobsohn E, et al. A randomized study in diabetic patients undergoing cardiac surgery comparing computer-guided glucose management with a standard sliding scale protocol. J Cardiothorac Vasc Anesth. 2008;22:377-82.

15. Roach GW, Kanchuger M, Mangano CM, Newman M, Nussmeier N, Wolman R, et al. Adverse cerebral outcomes after coronary bypass surgery. Multicenter Study of Perioperative Ischemia Research Group and the Ischemia Research and Education Foundation Investigators. N Engl J Med. 1996;335:1857-63.

16. Steel RGD, Torrie JH. Principles and procedures of statistics. A biometrical approach. 2nd ed. New York: McGraw-Hill; 1980.

17. D'Agostino RB Jr. Propensity scores in cardiovascular research. Circulation. 2007;115:2340-3.
18. Rosenbaum PR, Rubin DB. Reducing bias in observational studies using subclassification on the propensity score. J Am Stat Assoc. 1984;79:516-24.

19. Woodward M. Epidemiology: study design and data analysis. Boca Raton $(F L)$ : Chapman \& Hall/CRC. 1999;268-89.

20. D'Alessandro C, Leprince P, Golmard JL, Ouattara A, Aubert S, Pavie A, et al. Strict glycemic control reduces EuroSCORE expected mortality in diabetic patients undergoing myocardial revascularization. J Thorac Cardiovasc Surg. 2007; 134:29-37.

21. Hermanides J, Bosman RJ, Vriesendorp TM, Dotsch R, Rosendaal FR, Zandstra DF, et al. Hypoglycemia is associated with intensive care unit mortality. Crit Care Med. 2010;38:1430-4.

22. Van den Berghe G, Wouters P, Weekers F, Verwaest C, Bruyninckx F, Schetz M, et al. Intensive insulin therapy in the critically ill patients. $N$ Engl J Med. 2001; 345:1359-67.

23. Van den Berghe G, Wilmer A, Hermans G, Meersseman W, Wouters PJ, Milants I, et al. Intensive insulin therapy in the medical ICU. $N$ Engl J Med. 2006;354:449-61.

24. Arabi YM, Tamim HM, Rishu AH. Hypoglycemia with intensive insulin therapy in critically ill patients: predisposing factors and association with mortality. Crit Care Med. 2009;37:2536-44.

25. Wright RJ, Frier BM. Vascular disease and diabetes: is hypoglycaemia an aggravating factor? Diabetes Metab Res Rev. 2008;24:353-63.

26. Razavi Nematollahi L, Kitabchi AE, Stentz FB, Wan JY, Larijani BA, Tehrani MM, et al. Proinflammatory cytokines in response to insulin-induced hypoglycemic stress in healthy subjects. Metabolism. 2009;58:443-8.

27. Van den Berghe G, Wilmer A, Milants I, Wouters PJ, Bouckaert B, Bruyninckx F, et al. Intensive insulin therapy in mixed medical/surgical intensive care units: benefit versus harm. Diabetes. 2006;55:3151-9.

28. Stamou SC, Camp SL, Stiegel RM, Reames MK, Skipper E, Watts LT, et al. Quality improvement program decreases mortality after cardiac surgery. J Thorac Cardiovasc Surg. 2008;136:494-9.

\section{APPENDIX 1.}

Carolinas Medical Center (CMC)

Tight Blood Glucose Control Orders for Adult Patient in MICU, STICU, DHICU, CVRU, or NSICU (Target 80-120 gm/dL)

EndoTool Program

\section{CMC Medical Executive Committee}

Implement STANDING Physician order: SO CMC Tight Blood Glucose Control Orders for Adult Patient in MICU, STICU, DHICU, CVRU, or NSICU

Verify Allergies

Inclusion Criteria: Adult patient in MICU, STICU, DHICU, CVRU, or NSICU who meets clinical criteria below for known diabetic or nondiabetic patient and is receiving steady nutritional support. This nutrition can be steady IVs with dextrose solutions, steady tube feedings, or TPN.

Exclusion Criteria: Do not use this order set to manage a patient with diabetic ketoacidosis (DKA) unless approved by the attending physician. Contact MD for alternative blood glucose control measures if patient is eating or receiving intermittent tube feedings.

\section{Pharmacy}

250 units regular insulin in $250 \mathrm{~mL}$ normal saline (NS)

Dextrose 50\% IV PRN per EndoTool Program

Note: Mix all IV medications in NS or $1 / 2$ NS when possible

If an IV insulin infusion is started for tight glucose control: discontinue all current insulin orders, including PRN, sliding scale, continuous infusion, and/or scheduled bolus doses.

\section{Nursing}

Check blood glucose via finger stick or laboratory (BMP, CMP, or glucose) upon arrival to unit and PRN for symptoms and/or changes in vital signs, neurologic status, or nutrition status. 
For known Diabetic Patient:

(a) If glucose is greater than $130 \mathrm{gm} / \mathrm{dL}$ : initiate insulin infusion according to Insulin Infusion Orders below

(b) If glucose is $130 \mathrm{gm} / \mathrm{dL}$ or less: check glucose Q6H. Initiate Insulin Infusion Orders below if any blood glucose is greater than $130 \mathrm{gm} / \mathrm{dL}$.

For Nondiabetic Patient:

(a) If glucose is greater than $150 \mathrm{gm} / \mathrm{dL}$ : initiate insulin infusion according to Insulin Infusion Orders below.

(b) If glucose is $150 \mathrm{gm} / \mathrm{dL}$ or less: check glucose every AM. Initiate Insulin Infusion Orders below if any blood glucose is greater than $150 \mathrm{gm} / \mathrm{dL}$.

When blood glucose is stable for 24 hours, request specific insulin order from physician

Prior to starting the infusion, waste approximately $30 \mathrm{~mL}$ of the insulin mixture through the IV tubing to saturate the plastic tubing with insulin.

\section{INSULIN INFUSION ORDERS}

The patient must be on steady nutritional support to be eligible for EndoTool insulin dose calculations. This nutrition can be steady IVs with dextrose solutions, steady tube feedings, or TPN. Alternative blood glucose control measures must be used if the patient is eating or receiving intermittent tube feedings. Titration of the TPN or titration of the tube feeding or titration of clear liquids is allowed with the EndoTool protocol.

Start the EndoTool Program by entering the necessary patient information into the computer.

If patient is currently on the EndoTool Program and is transferring from another intensive care unit: enter the "BR" and "f $f$ " factors if known.

If patient is transferring from a nonintensive care unit: search for the patient by medical record number in the inactive file by clicking on the View inactive patient's link under the System Tasks heading.

Follow EndoTool Program instructions for insulin infusion rates, bolus doses, $\mathrm{D}_{50} \mathrm{~W}$ doses, and blood glucose determination frequency. For glucose levels of $80 \mathrm{mg} / \mathrm{dL}$ or less: the insulin infusion is zero (0.0) or OFF.

Nurse must obtain a physician order to give any dose that is not the calculated dose.

Use a micropump for insulin infusion and attach into a "Y" site as close to where the IV is inserted into the patient as possible with compatible IV fluids that infuse at a constant rate of at least $30 \mathrm{~mL} / \mathrm{h}$.

Insert Daily Summary Report and Order Sheet generated by EndoTool Program each morning into the patient's chart for documentation of the glucose readings and insulin doses administered.

If the patient is on TPN or tube feeding that is interrupted for more than 30 minutes while on the insulin infusion: run $\mathrm{D}_{10} \mathrm{~W}$ IV at the same rate as the interrupted TPN or tube feeding rate. (Must have OK from Neurosurgery if Neurosurgery is attending or consulting). Check glucose Q1H after a change in nutrition status and PRN.

\section{STOPPING EndoTool PROGRAM}

Discontinue EndoTool Program once the patient is allowed to eat and contact the primary physician regarding further orders for blood glucose management.

Patient on EndoTool program who has resumed oral intake: Print the Patient-specific SubQ insulin orders from EndoTool for SubQ insulin coverage. Leave IV insulin drip running for 2 hours after first dose of SubQ lantus insulin given.

Stop insulin drip once alternative diabetic treatment is ordered and started.

"IV Insulin Drip Orders" option may be printed with a physician order and used if the patient is ordered to remain on an insulin drip and is being transferred to another critical care unit.

Print the last MAR/Orders from the "Print Patient Reports/Orders" option and place in the patient's chart.

If the patient is transferring out of current room: click on the Patient Information tab and change the patient's status to inactive" by changing the "Active" status from "Y" to "N." Click "Save \& Continue" to save the information.

DATE: _ TIME: _ RN SIGNATURE:

(RN implementing Standing order)

DATE: TIME: — MD SIGNATURE:

Official Use only: Approved by CMC Medical Executive Committee(s) Approval Date: (May 2008) 